\title{
A note on observability tests for general polynomial and simple Wiener-Hammerstein systems ${ }^{1}$
}

\author{
Dragan Nešić * \\ CESAME, Batiment Euler, Avenue G. Lemaitre 4, 1348 Louvain la Neuve, Belgium
}

Received 22 August 1997; received in revised form 24 January 1998; accepted 11 June 1998

\begin{abstract}
We propose an algorithm, based on symbolic computation packages, for testing observability conditions of general polynomial systems, which were formulated in Sontag, SIAM J. Control Optim. 17 (1979) 139-151. Computational complexity of the observability test can be reduced and the test simplified for classes of polynomial systems. We illustrate this by considering the class of simple Wiener-Hammerstein systems, which consist of a series of two linear dynamic blocks between which a static nonlinearity is "sandwiched". We consider the case when the nonlinearity is a monomial $N(\cdot)=(\cdot)^{q}, q>1$. Simple necessary and sufficient conditions for observability are given and they resemble, but are subtly different from, the well known result on observability for the series connection of two linear systems. (C) 1998 Elsevier Science B.V. All rights reserved.
\end{abstract}

Keywords: Observability; Discrete-time; Wiener-Hammerstein systems; Polynomial systems; Symbolic computation

\section{Introduction}

Observability is one of the fundamental notions in control theory. Our ability to reconstruct, and not directly measure, the state vector of the system is not only theoretically appealing but also of utmost importance in practice. Indeed, if the plant is observable, the number of (expensive) sensors necessary for operation of the system can be drastically reduced. This implication of the observability notion is most obvious one. However, a number of other important analysis and synthesis control questions directly depend on this notion. For instance, observability plays a prominent role in realization theory; in optimal control theory

\footnotetext{
* Tel: 0104780 41; e-mail: nesic@auto.ucl.ac.be.

${ }^{1}$ This paper presents research results of the Belgian Programme on Inter-university Poles of Attraction, initiated by the Belgian State, Prime Minister's Office for Science, technology and Culture. The scientific responsibility rests with its author.
}

(LQ), stability of optimal regulators depend directly on whether the unstable states are "observable" in the cost, etc.

Observability of polynomial discrete-time systems was studied in $[15,16]$, where Sontag defined and analyzed relationships between a number of observability properties. The conditions for multiple experiment observability which he presented are difficult to check and they amount to testing if a (very general) polynomial mapping is one-to-one. No general method was provided in [16] for testing the bijectivity and only methods which provide sufficient conditions were referred to. We use constructions from $[16,17]$ and recently emerged symbolic computation packages (Gröbner basis method [4] and QEPCAD [2]) as tools in order to automate testing of the observability for general polynomial systems. The algorithmic test is guaranteed to check necessary and sufficient conditions for observability in finite time. 


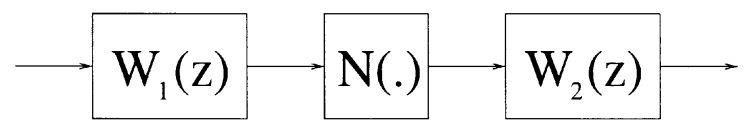

Fig. 1. Simple Wiener-Hammerstein model.

In the second part of the paper we concentrate on a particular class of polynomial systems, often referred to as simple Wiener-Hammerstein systems $[5,6]$. Simple Wiener-Hammerstein systems arise in black-box identification for nonlinear systems [5, 6] (see Fig. 1). These systems can be viewed as a series connection of two linear systems between which there is a static nonlinearity, often a polynomial. We concentrate on the situation when the nonlinearity is a monomial $N(\cdot)=(\cdot)^{q}, q>1, q \in \mathbb{N}$.

If we view a simple Wiener-Hammerstein system as a series connection of a linear system with output nonlinearity (called also output nonlinear or simple Wiener model) and another linear system, we can state a very elegant observability test which resembles the well-known observability condition for purely linear series. In the linear case, besides observability of the linear subsystems, the set of poles of the first subsystem and the set of zeroes of the second subsystem must have an empty intersection in order to have observability of the overall system [7]. For simple Wiener-Hammerstein systems, however, besides observability of the subsystems, the set of poles of the first linear block and the set of zeroes of the second linear block must not have the common zero element. In other words, any pole/zero cancellation is allowed except for the case when the first linear block has a pole and the second subsystem has a zero at the origin.

This resembles very much the statement obtained in [8-10] for series connection of a sign linear system with another linear system (in Fig. 1, the nonlinearity is $N(\cdot)=\operatorname{sign}(\cdot)$ ). There, however, observability of subsystems always implies observability of the connection, which is not case for the systems we consider. On the other hand, the result presented in [8] on simple Wiener-Hammerstein systems with saturating nonlinearities (Fig. 1 with $N(\cdot)=\operatorname{sat}(\cdot)$ ) excludes any pole/zero cancelation of the linear blocks. The result we obtain for simple Wiener-Hammerstein systems is necessary and sufficient and the situation is interesting since only some pole/zero cancelations destroy observability.

Let us finally remark that a number of controllability results for generalized Hammerstein and simple Wiener-Hammerstein systems was obtained in
[11-14], where similar conditions for null and complete controllability were obtained. In particular, complete controllability results in $[13,12]$ can be regarded as "dual" to the observability result obtained in this paper, although the proofs are quite different.

The paper is organized as follows. In Section 2 we present definitions and preliminaries. In Section 3 an observability test for general polynomial systems which is based on the Gröbner basis method is presented. Section 4 is dedicated to observability of simple Wiener-Hammerstein systems and in the last section we summarize our results.

\section{Preliminaries}

Consider general polynomial systems of the form

$$
\begin{aligned}
& x(k+1)=f(x(k), u(k)), \\
& y(k)=h(x(k)),
\end{aligned}
$$

where $y \in \mathbb{R}^{p}, \quad u \in \mathbb{R}^{m}, x \in \mathbb{R}^{n}$ and $h$ and $f$ are vector polynomial functions in all their arguments. We denote the output of the system (1) at time step $N$ which is obtained when a control sequence $U_{N}=\{u(0), u(1), \ldots, u(N-1)\}$ is applied to the system and emanating from the initial state $x$ as $y\left(N, x, U_{N}\right)$.

The notion of observability that we investigate is:

Definition 1. System (1) is observable if for each pair of initial states $\xi \neq \eta$, there exists an integer $N$ and an input sequence $U_{N}$ which yields $y\left(N, \xi, U_{N}\right) \neq y\left(N, \eta, U_{N}\right)$.

Observability in the sense of Definition 1 represents the so-called multiple experiment observability - any two pairs of states can be distinguished by some input sequence. A number of other observability properties were defined and their relationships for polynomial systems analyzed in [16].

We use the standard definitions of rings and fields [4]. The sets of real, natural and rational numbers are respectively denoted as $\mathbb{R}, \mathbb{N}$ and $\mathbb{Q} . \mathbb{R}^{n}$ is a set of all $n$-tuples of elements of $\mathbb{R}$, where $n \in \mathbb{N}$. The ring of polynomials in $n$ variables over a field $k$ is denoted as $k\left[x_{1}, x_{2}, \ldots, x_{n}\right]$.

Let $f_{1}, f_{2}, \ldots, f_{s}$ be polynomials in $\mathbb{R}\left[x_{1}, x_{2}, \ldots, x_{n}\right]$. Then we define $V\left(f_{1}, f_{2}, \ldots, f_{s}\right)=\left\{\left(a_{1}, a_{2}, \ldots, a_{n}\right) \in\right.$ $\mathbb{R}^{n}: f_{i}\left(a_{1}, a_{2}, \ldots, a_{n}\right)=0$ for all $\left.1 \leqslant i \leqslant s\right\}$. We call $V\left(f_{1}, f_{2}, \ldots, f_{s}\right)$ the real algebraic set or real variety 
defined by the polynomials $f_{1}, f_{2}, \ldots, f_{s}$. A subset $I \subset \mathbb{R}\left[x_{1}, x_{2}, \ldots, x_{n}\right]$ is an ideal if: $0 \in I ; f, g \in I$, then $f+g \in I ; f \in I$ and $h \in \mathbb{R}\left[x_{1}, \ldots, x_{n}\right]$, then $h f \in I$. Let $f_{1}, f_{2}, \ldots, f_{s}$ be polynomials in $\mathbb{R}\left[x_{1}, x_{2}, \ldots, x_{n}\right]$. Then the set $\left\langle f_{1}, \ldots, f_{s}\right\rangle$ defined as

$$
\left\langle f_{1}, \ldots, f_{s}\right\rangle=\left\{\sum_{i=1}^{s} h_{i} f_{i}: h_{1}, \ldots, h_{s} \in \mathbb{R}\left[x_{1}, \ldots, x_{n}\right]\right\}
$$

is called the ideal generated by $f_{1}, f_{2}, \ldots, f_{s}$. We use the notation $V(J)$ to denote the variety $V\left(f_{1}, \ldots, f_{n}\right)$, where $f_{i}$ are the generators of the ideal $J$.

We use the symbolic computation packages: the Gröbner basis algorithm [1, 4] and QEPCAD [2, 3]. For space reasons, we do not present any details on the algorithms and the reader is referred to $[1,4,2,3]$ for the description of the operation of algorithms.

A set $S \subset \mathbb{R}^{n}$ is called semi-algebraic if it can be constructed by finitely many applications of union, intersection and complementation operations on sets of the form $\left\{x \in \mathbb{R}^{n}: f_{i}(x) \geqslant 0\right\}$, where $f_{i}$ are polynomials in $x$ with real coefficients. Given a semi-algebraic set $S$ we denote its defining expression as $S(x)$. Given a set of polynomials $f_{1}, \ldots, f_{s}$, their reduced Gröbner basis is denoted as $G B\left[f_{1}, \ldots, f_{s}\right]$ [4].

\section{An algorithm for observability testing}

The construction given below is presented for SISO systems (1) to simplify presentation but it can be used for general MIMO case. The method that we use below is used in $[16,17]$ to prove some results. However, in [16] the test for checking observability notion of Definition 1 requires testing if a polynomial mapping is bijective, which is not easy and only sufficient conditions for this were referred to in the paper. By using the Gröbner basis method and QEPCAD we gain a straightforward algorithmic observability test, which tests necessary and sufficient observability conditions. The algorithm is guaranteed to test this notion in finite time.

We consider all states $\xi, \eta \in \mathbb{R}^{n}, \xi \neq \eta$ which produce the same output sequence irrespective of the applied input sequence. A real variety $V_{z} \subset \mathbb{R}^{n} \times \mathbb{R}^{n}$ is constructed in the following way (see also [17]).

If two states $\xi, \eta \in \mathbb{R}^{n}$ cannot be distinguished by any input sequence, then necessarily we have that $h(\xi)=h(\eta)$. We construct an ideal $J_{1}=\langle h(\xi)-h(\eta)\rangle$. Suppose that $h(f(x, u))$ depends explicitly on $u$. We can write $h(f(x, u))=h_{m}(x) u^{m}+\cdots+h_{1}(x) u+h_{0}(x)$.
Then the states $\xi, \eta$ which produce the same output in first and second time steps irrespective of the applied control satisfy

$$
\begin{aligned}
& h(\xi)=h(\eta), \\
& h_{i}(\xi)=h_{i}(\eta), \quad \forall i=0,1, \ldots, m .
\end{aligned}
$$

We construct an ideal $J_{2}=\left\langle h(\xi)-h(\eta), h_{m}(\xi)-\right.$ $\left.h_{m}(\eta), \ldots, h_{0}(\xi)-h_{0}(\eta)\right\rangle$. Notice, that $J_{1} \subseteq J_{2}$ by construction. If $J_{1}=J_{2}$, then all states that cannot be distinguished by the inputs are contained in the variety $V\left(J_{1}\right) \subset \mathbb{R}^{n} \times \mathbb{R}^{n}$. Suppose that $J_{1} \neq J_{2}$. Then we have that $J_{1} \subset J_{2}$. Let us compute the set of states for which the outputs are identical for the first three time steps. These states necessarily satisfy $h_{i}(f(\xi, u))-h_{i}(f(\eta, u))=0, \quad \forall u \in \mathbb{R}, \quad \forall i=0,1, \ldots$, $m_{0}$. If we denote $h_{i}(f(x, u))=h_{p_{i}, i}(x) u^{p_{i}}+\cdots+$ $h_{1, i}(x) u+h_{0, i}(x)$, we construct the ideal

$$
\begin{gathered}
J_{3}=\left\langle h(\xi)-h(\eta), h_{m}(\xi)-h_{m}(\eta), \ldots, h_{0}(\xi)-h_{0}(\eta),\right. \\
\left.h_{p_{m}, m}(\xi)-h_{p_{m}, m}(\eta), \ldots, h_{0,0}(\xi)-h_{0,0}(\eta)\right\rangle .
\end{gathered}
$$

If $J_{3}=J_{2}$ the variety $V\left(J_{2}\right)$ contains all indistinguishable states. Otherwise, we have $J_{1} \subset J_{2} \subset J_{3}$. We continue the same construction to obtain an ascending chain of ideals $J_{1} \subset J_{2} \subset J_{3} \subset \cdots$, which must terminate $[17,4]$, that is $J_{N}=J_{N+1}$ for some $N$. We let $V_{z}=V\left(J_{N}\right)$. All the indistinguishable states belong to the set $S_{z}=V_{z} \cap\{(\xi, \eta): \xi \neq \eta\}$. This set is a semialgebraic subset of $\mathbb{R}^{n} \times \mathbb{R}^{n}$. If we denote the set of equations obtained when all generators of $J_{N}$ are set equal to zero as $J_{N}(\xi, \eta)$, using QEPCAD we can check the following decision problem:

$(\exists \xi)(\exists \eta)\left[J_{N}(\xi, \eta) \wedge \xi \neq \eta\right]$.

If the answer to Eq. (3) is FALSE $\left(S_{z}=\emptyset\right)$, the systems observable and vice versa. We can compare whether the ideals $J_{i}$ and $J_{i+1}, \forall i$ are equal by comparing their reduced Gröbner bases for the same ordering. The construction which was just described can be formally stated as an algorithmic observability test, which stops in finite time:

\section{An algorithmic observability test:}

1. $k=1, f(x, u), h(x), J_{1}=\langle h(\xi)-h(\eta)\rangle, \quad G_{1}=\mathrm{GB}$ $\left[J_{1}\right]$, fix a monomial ordering.

2. $k=k+1$.

3. Compute ideal $J_{k}$ and its reduced Gröbner basis $G_{k}=\mathrm{GB}\left[J_{k}\right]$.

4. Compare whether $G_{k}=G_{k-1}$. If yes go to 5. If not go to 2 . 
5. Check using QEPCAD if the decision problem (3) is satisfied. If not, the system is observable and vice versa.

The observability test for polynomial systems terminates always in finite time, since we need to compute finitely many times the Gröbner basis of certain ideals and then use QEPCAD to solve the decision problem (3). The computational cost associated with the observability test is large.

Sometimes we may not have to use the QEPCAD algorithm in testing observability. From construction of $V_{z}=V\left(J_{N}\right)$, we see that $V(\xi-\eta) \subseteq V_{z}$ and the system is observable if and only if $V_{z}=V(\xi-\eta)$. If at one step we obtain that the reduced Gröbner basis of the ideal $J_{k}$ is $\{\xi-\eta\}$, we know that the system is observable since it immediately follows that $J_{k}=J_{k+1}$ and $V_{z}=V(\xi-\eta)$. This situation is illustrated by the following example.

Example 1. Consider the simple Wiener system

$x_{1}(k+1)=x_{2}(k)$,

$x_{2}(k+1)=-x_{1}(k)-2 x_{2}(k)+u(k)$,

$y(k)=x_{1}^{2}(k)$.

The system consists of a linear dynamical block and quadratic static nonlinearity, which is at the output of the system.

To find the variety $V_{z} \subset \mathbb{R}^{n} \times \mathbb{R}^{n}$, the following ideals are constructed:

$$
\begin{aligned}
J_{1}= & \left\langle\eta_{1}^{2}-\xi_{1}^{2}\right\rangle, \\
J_{2}= & \left\langle\eta_{1}^{2}-\xi_{1}^{2}, \eta_{2}^{2}-\xi_{2}^{2}\right\rangle, \\
J_{3}=\left\langle\eta_{1}^{2}-\xi_{1}^{2}, \eta_{2}^{2}-\xi_{2}^{2},\left(\eta_{1}+2 \eta_{2}\right)^{2}-\left(\xi_{1}+2 \xi_{2}\right)^{2},\right. & \left.\left(\eta_{1}+2 \eta_{2}\right)-\left(\xi_{1}+2 \xi_{2}\right)\right\rangle, \\
J_{4}=\left\langle\eta_{1}^{2}-\xi_{1}^{2}, \eta_{2}^{2}-\xi_{2}^{2},\left(\eta_{1}+2 \eta_{2}\right)^{2}-\left(\xi_{1}+2 \xi_{2}\right)^{2},\right. & \left(\eta_{1}+2 \eta_{2}\right)-\left(\xi_{1}+2 \xi_{2}\right)\left(2 \eta_{1}+\eta_{2}\right)^{2} \\
& -\left(2 \xi_{1}+\xi_{2}\right)^{2}, 2 \eta_{1}+\eta_{2}-2 \xi_{1}-\xi_{2}-2 \eta_{1} \\
& \left.-3 \eta_{2}+2 \xi_{1}+3 \xi_{2}\right\rangle
\end{aligned}
$$

and by using the Gröbner basis algorithm, with lexicographic ordering $\xi_{1} \succ \xi_{2} \succ \eta_{1} \succ \eta_{2}$ we obtain that $\mathrm{GB}\left[J_{4}\right]=\left\{\eta_{1}-\xi_{1}, \eta_{2}-\xi_{2}\right\}$ and therefore $J_{4}=J_{5}$. The system is observable since $V_{z}=V\left(J_{4}\right)=V\left(\eta_{1}-\right.$ $\left.\xi_{1}, \eta_{2}-\xi_{2}\right)$ and consequently $S_{z}=\emptyset$.

\section{Simple Wiener-Hammerstein systems}

In this section we concentrate on simple WienerHammerstein systems of the form (see Fig. 1)

$x_{1}(k+1)=A x_{1}(k)+b u(k)$,

$x_{2}(k+1)=F x_{2}(k)+g\left(c x_{1}(k)\right)^{q}$,

$y(k)=h x_{2}(k)$,

where $x_{i} \in \mathbb{R}^{n_{i}}, i=1,2, n_{1}+n_{2}=n, \quad u \in \mathbb{R}, \quad q \in \mathbb{N}$, $q>1$ and matrices $A, F, b, g$ are of appropriate dimensions. The system consists of a series connection of two linear dynamical blocks

$$
\begin{aligned}
& W_{1}(z)=\frac{y_{1}(z)}{u_{1}(z)}=c(z I-A)^{-1} b=\frac{\beta_{1}(z)}{\alpha_{1}(z)}, \\
& W_{2}(z)=\frac{y_{2}(z)}{u_{2}(z)}=h(z I-F)^{-1} g=\frac{\beta_{2}(z)}{\alpha_{2}(z)},
\end{aligned}
$$

interconnected via the static nonlinearity $u_{2}(k)=$ $\left(y_{1}(k)\right)^{q}$. We also have that the input of the overall system $u(k)=u_{1}(k)$ and output of the overall system $y(k)=y_{2}(k)$. To simplify exposition, we assume without loss of generality that there are no feed-through terms for transfer functions $W_{i}(z)$. We emphasize, however, that our main results hold in this case. It is also assumed that the relative degree $d$ of the system (6) is finite. In other words, the control explicitly appears in the formula for $y\left(k, x, U_{k}\right)$ for some $k<n$. This implies that $b \neq 0$ and $g \neq 0$.

The following decomposition of the simple WienerHammerstein system is used in the sequel. We say that the linear dynamical block $W_{1}(z)$ and the static nonlinearity $(\cdot)^{q}$ is the subsystem $S_{1}$

$$
\begin{aligned}
& x_{1}(k+1)=A x_{1}(k)+b u(k), \\
& z(k)=\left(y_{1}(k)\right)^{q}=\left(c x_{1}(k)\right)^{q}
\end{aligned}
$$

and that the linear block $W_{2}(z)$ is the subsystem $S_{2}$

$$
\begin{aligned}
& x_{2}(k+1)=F x_{2}(k)+g u(k), \\
& y(k)=h x_{2}(k) .
\end{aligned}
$$

Notice that only the first subsystem (8) is nonlinear in output (simple Wiener model), whereas subsystem (9) is linear. The well-known condition for observability of linear system (9) is that the observability matrix

$$
\left(\begin{array}{c}
h \\
h F \\
\cdots \\
h F^{n-1}
\end{array}\right)
$$


is nonsingular and then we say that the pair $(h, F)$ is an observable pair. An obvious necessary condition for observability of system $(8)$ is that $(c, A)$ is an observable pair. If $q$ in Eq. (8) is an even integer, the output nonlinearity is not bijective and we also have the following necessary condition for observability, which was proved in $[8,9]$ (Lemma 2.4 in [9]):

Lemma 1. System (8) with $q$ an even integer is observable only if $A$ is nonsingular.

We state the necessary and sufficient conditions for observability of simple Wiener system (8) with $q$ an even integer:

Theorem 1. Simple Wiener system (8), with q a positive even integer, is observable if and only if

1. $(c, A)$ is an observable pair,

2. $A$ is non-singular,

3. $b \neq 0$.

To shorten notation, we write $z(k)=z_{k}, u(k)=u_{k}$ and $x_{1}(0)=x_{1}$.

Proof of Theorem 1. (Necessity) The necessity of the first condition is obvious. Lemma 1 tells us that the second condition is necessary. We show that $b \neq 0$ is also necessary. Suppose that system (8) is observable but $b=0$. The output of system (8) at time steps $k=0,1,2, \ldots$ for any sequence of controls $U_{k}$ is $z(k)=\left(c A^{k-1} x_{1}\right)^{q}, k=0,1, \ldots$. It is easy to see now that the states $\eta_{1}=-\xi_{1}, \xi_{1} \neq 0$ are indistinguishable since $\left(c A^{k} \eta_{1}\right)^{q}=\left(-c A^{k} \eta_{1}\right)^{q}, \forall k=0,1, \ldots$, which contradicts our assumption on observability.

(Sufficiency) Suppose that the conditions of Theorem 1 are satisfied but system (8) is not observable. Consider the outputs $z$ of system (8) with the control sequence $U_{k}$ :

$z\left(k, x_{1}, U_{k}\right)=\left(c A^{k} x_{1}\right)^{q}, \quad k=0,1, \ldots, d_{1}-2$,

$z\left(d_{1}, x_{1}, U_{d_{1}}\right)=\left(c A^{d_{1}} x_{1}+c A^{d_{1}-1} b u_{0}\right)^{q}$,

$z\left(d_{2}, x_{1}, U_{d_{1}+1}\right)=\left(c A^{d_{1}+1} x_{1}+c A^{d_{1}} b u_{0}+c A^{d_{1}-1} b u_{1}\right)^{q}$,

Notice that because of observability of $(c, A)$, we have that $c A^{d_{1}-1} b \neq 0$ for some $d_{1} \in\left\{1, \ldots, n_{1}\right\}$ since $b \neq 0$ and hence system (8) has a finite relative degree $d_{1} \leqslant n_{1}$. Hence, $c A^{d_{1}-1} b \neq 0$. We note that any two indistinguishable states $\xi_{1} \neq \eta_{1}$ should satisfy $\forall k, \forall U_{k}, z\left(k, \eta_{1}, U_{k}\right)=z\left(k, \xi_{1}, U_{k}\right)$. For $k=d_{1}$, we obtain that

$$
\left(c A^{d_{1}} \eta_{1}+c A^{d_{1}-1} b u_{0}\right)^{q}-\left(c A^{d_{1}} \xi_{1}+c A^{d_{1}-1} b u_{0}\right)^{q} \equiv 0,
$$

as a function of $u_{0}$. By using the binomial formula, we can write the above expression as a polynomial in $u_{0}$ whose coefficients are polynomials in entries of $\eta_{1}$ and $\xi_{1}$. Then expression (10) is identically equal to zero if and only if all coefficients of the polynomial are equal to zero. Consider the coefficient that multiplies $u_{0}^{q-1}$ :

$\left(\begin{array}{l}q \\ 1\end{array}\right)\left(c A^{d_{1}-1} b\right)^{q-1}\left(c A^{d_{1}} \eta_{1}-c A^{d_{1}} \xi_{1}\right)$,

where

$\left(\begin{array}{l}q \\ j\end{array}\right)=\frac{q !}{j !(q-j)}, \quad j=0,1, \ldots, q$.

Since $c A^{d_{1}-1} b \neq 0$ we necessarily have that indistinguishable states must satisfy $c A^{d_{1}} \eta_{1}-c A^{d_{1}} \xi_{1}=0$. Similarly, by looking at expressions $z\left(k, \eta_{1}, U_{k}\right)-z(k$, $\left.\xi_{1}, U_{k}\right) \equiv 0, k=d_{1}+1, \ldots, d_{1}+n_{1}-1$, we obtain that indistinguishable states must satisfy $c A^{d_{1}+k} \eta_{1}-c A^{d_{1}+k}$ $\xi_{1}=0, k=1,2, \ldots, n_{1}-1$. We can write these equations in the matrix form:

$$
\left(\begin{array}{c}
c \\
c A \\
\cdots \\
c A^{n_{1}-1}
\end{array}\right) A^{d_{1}}\left(\eta_{1}-\xi_{1}\right)=0 .
$$

Since $(c, A)$ is an observable pair and $A$ is nonsingular ( $A^{d_{1}}$ is also nonsingular) we have that Eq. (11) holds if and only if $\eta_{1}=\xi_{1}$ and hence there do not exist indistinguishable states $\eta_{1} \neq \xi_{1}$, a contradiction.

We denote the sets of poles of the linear blocks (7) as $\mathscr{P}_{i}=\left\{z \in \mathbb{C}: a_{i}(z)=0\right\}, i=1,2$ and the sets of zeros as $\mathscr{Z}_{i}=\left\{z \in \mathbb{C}: b_{i}(z)=0\right\}, i=1,2$. In order to appreciate the subtle difference of our result from the linear result, we present the well known linear observability condition for observability of a series connection of two systems.

Theorem 2 (Kailath [7]). The linear system (6), with $q=1$, is observable if and only if both subsystems (8) and (9) are observable and $\mathscr{P}_{1} \cap \mathscr{Z}_{2}=\emptyset$. 
Now we state the main result of this section (notice the difference with Theorem 2).

Theorem 3. The simple Wiener-Hammerstein system (6), with $q>1$, is observable if and only if subsystems (8) and (9) are both observable and $0 \notin \mathscr{P}_{1} \cap \mathscr{Z}_{2}$.

Comment 1. It is interesting to explore the subtle difference between the observability conditions for the linear case in Theorems 2 and 3. If we connect two observable linear systems in series, any pole zero cancelation $\left(\mathscr{P}_{1} \cap \mathscr{Z}_{2} \neq \emptyset\right)$ leads to loss of observability. If we connect an observable simple Wiener system in series with an observable linear system, only pole of $W_{1}(z)$ and zero of $W_{2}(z)$ at the origin $\left(0 \in \mathscr{P}_{1} \cap \mathscr{Z}_{2}\right)$ destroys observability. An interesting interpretation of our result is as follows. Suppose that $W_{1}(z)$ has no poles at the origin, $W_{1}(z)$ and $W_{2}(z)$ are both observable but the linear series connection is not (hence $\mathscr{P}_{1} \cap \mathscr{Z}_{2} \neq \emptyset$ ). By nonlinearizing the interconnection channel (inserting, for example, a cubic nonlinearity between the linear blocks) we recover observability.

Proof of Theorem 3. (Sufficiency) Consider first the situation when both $(c, A)$ and $(h, F)$ are observable and $0 \notin \mathscr{P}_{1}$, that is, the matrix $A$ is nonsingular. Consider the outputs of system (6) at time steps $k=0,1, \ldots$ as a function of past inputs and the initial state. To shorten notation, we write $y(k)=y_{k}, u(k)=u_{k}$ and $x_{i}(0)=x_{i}, i=1,2, \ldots, n$.

$y_{0}=h x_{2}$,

$y_{1}=h F x_{2}+h g\left(c x_{1}\right)^{q}$,

$y_{2}=h F^{2} x_{2}+h F g\left(c x_{1}\right)^{q}+h g\left(c A x_{1}+c b u_{0}\right)^{q}$

$$
\begin{gathered}
y_{k}=h\left(F^{k} x_{2}+\sum_{j=0}^{k-1} F^{k-1-j} g\left(C \left(A^{j} x_{1}\right.\right.\right. \\
\left.\left.\left.+\sum_{i=0}^{j-1} A^{j-1-i} b u_{i}\right)\right)^{q}\right)
\end{gathered}
$$

We would consider which polynomials in $x$ are used from equations (12) to form ideals $J_{k}$ in the previous section, which are used in the computation of the variety $V_{z} \subset \mathbb{R}^{n} \times \mathbb{R}^{n}$. The first two ideals are readily read out from the Eqs. (12), and they are given by

$$
\begin{aligned}
& J_{1}=\left\langle h \xi_{2}-h \eta_{2}\right\rangle, \\
& J_{2}=\left\langle h \xi_{2}-h \eta_{2}, h F \xi_{2}+h g\left(c \xi_{1}\right)^{q}-h F \eta_{2}+h g\left(c \eta_{1}\right)^{q}\right\rangle .
\end{aligned}
$$

To form the ideal $J_{3}$, consider the expression for $y_{2}$ in Eq. (12). Notice that using the binomial formula, we can write the 1.h.s. as follows:

$h F^{2} x_{2}+h F g\left(c x_{1}\right)^{q}+h g \sum_{j=0}^{q}\left(\begin{array}{l}q \\ j\end{array}\right)\left(c A x_{1}\right)^{q-j}\left(c b u_{0}\right)^{j}$.

Without loss of generality, we can assume that subsystems (8) and (9) have relative degrees 1, that is $h g \neq 0, c b \neq 0 .{ }^{2}$ Then, the ideal $J_{3}$ would necessarily have the following generators $\left(c A \xi_{1}\right)^{j}-\left(c A \eta_{1}\right)^{j}, j=$ $1, \ldots, q-1$. Similarly, it can be shown using the binomial expression for 1.h.s. of equations for $y_{k}, k \geqslant 4$ that the ideals $J_{k}, k \geqslant 4$ have necessarily generators of the form:

$\left(c A^{k-2} \xi_{1}\right)^{j}-\left(c A^{k-2} \eta_{1}\right)^{j}, \quad j=1, \ldots, q-1$.

Since the first subsystem is observable by assumption and $A$ is nonsingular, we can see from the ideal $J_{n+2}$ that

$\left(\begin{array}{c}c \\ c A \\ \cdots \\ c A^{n-1}\end{array}\right) A\left(\xi_{1}-\eta_{1}\right)=0$,

must be satisfied for any two states in order to be indistinguishable and this implies that any possible indistinguishable states must satisfy $\xi_{1}=\eta_{1}$, since $(c, A)$ is an observable pair. With this equation, we can considerably simplify the polynomials that define ideals $J_{k}$ since $\xi_{1}$ and $\eta_{1}$ "cancel each other out" and we obtain the simplified ideals $J_{k}=\left\langle h\left(\xi_{2}-\eta_{2}\right), \ldots, h F^{k-1}\left(\xi_{2}-\eta_{2}\right)\right\rangle, k=1, \ldots, n+2$. Hence indistinguishable states should also satisfy

$\left(\begin{array}{c}h \\ h F \\ \ldots \\ h F^{n}\end{array}\right)\left(\xi_{2}-\eta_{2}\right)=0$

\footnotetext{
${ }^{2}$ If this condition is violated, the proof follows along the same lines but we need to consider the formulas outputs for large enough time $k$, for which $h F^{i} g \neq, c A^{j} b \neq 0, i, j \in\{0,1,2, \ldots, k\}$. Since system (6) is assumed to have a finite relative degree, such a time always exists.
} 
and since the pair $(h, F)$ is observable, this can happen only when $\xi_{2}-\eta_{2}=0$. We have that $V_{z}=V(\xi-\eta)$, which implies $S_{z}=\emptyset$ and the system is observable. Notice that in the purely linear case the polynomials (14) do not appear since then $q-1=0$ and they are defined only for $q>1$ ! This shows the difference between the purely linear case and the nonlinear case that we consider.

We note that if $q$ is an even integer, then from Lemma 1 it follows that it is necessary for observability of the first subsystem that $0 \notin \mathscr{P}_{1}$. Hence, we only need to address the situation when $q$ is an odd integer and $0 \in \mathscr{P}_{1}$.

Suppose, therefore that $q$ is odd, $0 \in \mathscr{P}_{1}$ and $0 \notin \mathscr{Z}_{2}$. First notice that since $A$ is singular, we can always find a coordinate transformation for the first subsystem such that $T^{-1} A T=\operatorname{diag}\left\{A_{11}, J\right\}$ where $A_{11}$ is nonsingular and $J$ nilpotent. Suppose that the systems matrix $A$ is already in the diagonal form and decompose the state vector $x_{1}=\left(\begin{array}{ll}\bar{x}_{1}^{\mathrm{T}} & \hat{x}_{1}^{\mathrm{T}}\end{array}\right)^{\mathrm{T}}$ so that variables with the "bar" correspond to nonzero modes and with the "hat" to zero modes. We first show that it is possible to concentrate on showing indistinguishability only for states corresponding to zero modes.

Consider the output $y_{k}(12)$ for $k$ sufficiently large enough so that $c A^{k+1}=\left(\begin{array}{ll}\bar{c} & 0\end{array}\right)$, that is $J^{k+1}=0$. If we consider then the subsequent outputs, we obtain that the following equations should be satisfied for the indistinguishable states:

$\left(\bar{c} A_{11}^{k-2} \bar{\xi}_{1}\right)^{j}-\left(\bar{c} A_{11}^{k-2} \bar{\eta}_{1}\right)^{j}=0, \quad j=1, \ldots, q-1$

and because of observability of $(c, A)$ we obtain that $\bar{\xi}_{1}=\bar{\eta}_{1}$. So we can concentrate only on the situation when the matrix $A=J$ is nilpotent.

In order to simplify presentation, the rest of the proof is carried out for a special situation when $\alpha_{1}(z)=z^{2}, \quad \beta_{1}(z)=c_{2} z+c_{1}, \quad \alpha_{2}(z)=z^{3}+a_{1} z^{2}+a_{2} z$ $+a_{3}, \beta_{2}(z)=b_{1} z^{2}+b_{2} z+b_{3}$. The following formulas for Markov parameters as functions of coefficients of $\beta_{2}(z)$ and $\alpha_{2}(z)$ of the second subsystem are used:

$h g=b_{1}$,

$h F g=-a_{1} b_{1}+b_{2}$,

$h F^{2} g=-a_{1}\left(-a_{1} b_{1}+b_{2}\right)-a_{2} b_{1}+b_{3}$,

$h F^{k} g=-a_{1}\left(h F^{k-1} g\right)-a_{2}\left(h F^{k-2} g\right)$

$-a_{3}\left(h F^{k-3} g\right), \quad k \geqslant 3$.

For any two indistinguishable states $\xi \neq \eta$ we should have that $\forall k, \forall U_{k}, y\left(k, \xi, U_{k}\right)=y\left(k, \eta, U_{k}\right)$. But then for indistinguishable states it holds also that for any $t_{i} \in \mathbb{R}, N \in \mathbb{N}$

$\sum_{i=1}^{N} t_{i} y\left(k_{i}, \xi, U_{k_{i}}\right)=\sum_{i=1}^{N} t_{i} y\left(k_{i}, \eta, U_{k_{i}}\right), \quad \forall U_{k_{i}}$.

We can simplify the formulas that define ideals $J_{k}$ considerably if instead of formulas for $y_{k}, k \geqslant 3$ (12), we consider the following sums $y_{k}+a_{1} y_{k-1}+a_{2} y_{k-2}+$ $a_{3} y_{k-3}, k \geqslant 3$, where $a_{i}$ are coefficients of the polynomial $\alpha_{2}(z)$. By using, formulas for Markov parameters (17) we obtain

$$
\begin{aligned}
y_{0}= & h x_{2}, \\
y_{1}= & h F x_{2}+h g\left(c x_{1}\right)^{q}, \\
y_{2}= & h F^{2} x_{2}+h F g\left(c x_{1}\right)^{q}+h g\left(c J x_{1}+c b u_{0}\right)^{q}, \\
y_{3}+ & a_{1} y_{2}+a_{2} y_{1}+a_{3} y_{0} \\
= & b_{3}\left(c x_{1}\right)^{q}+b_{2}\left(c J x_{1}+c b u_{0}\right)^{q}+b_{1}\left(c J b u_{0}+c b u_{1}\right)^{q}, \\
y_{4}+ & a_{1} y_{3}+a_{2} y_{2}+a_{3} y_{1} \\
= & b_{3}\left(c J x_{1}+c b u_{0}\right)^{q}+b_{2}\left(c J b u_{0}+c b u_{1}\right)^{q} \\
& +b_{1}\left(c J b u_{1}+c b u_{2}\right)^{q}, \\
y_{5}+ & a_{1} y_{4}+a_{2} y_{3}+a_{3} y_{2} \\
= & b_{3}\left(c J b u_{0}+c b u_{1}\right)^{q}+b_{2}\left(c J b u_{1}+c b u_{2}\right)^{q} \\
& +b_{1}\left(c J b u_{2}+c b u_{3}\right)^{q} .
\end{aligned}
$$

Eq. (17) shows that due to the finite impulse response of the first subsystem, we can compute all indistinguishable states only from the first five Eq. (17), that is using $y_{i}, i=0, \ldots, 4$. Since, by assumption the polynomial $\beta_{2}(z)$ has no zero roots, we have that $b_{3} \neq 0$. From the fifth formula in Eq. (17) we see that the indistinguishable states should necessarily satisfy $\left(c J \xi_{1}\right)^{j}-\left(c J \eta_{1}\right)^{j}=0, j=1, \ldots, q$. From the fourth equation, we then also have that $\left(c \xi_{1}\right)^{q}-\left(c \eta_{1}\right)^{q}=0$ and since $q$ is odd, this implies that

$\left(\begin{array}{c}c \\ c J\end{array}\right)\left(\xi_{1}-\eta_{1}\right)=0$

which because of observability of $(c, A)=(c, J)$ implies that for indistinguishable states we have $\xi_{1}=\eta_{1}$. Using this formula, from the first three equations in Eq. (17) we then obtain that

$\left(\begin{array}{c}h \\ h F \\ h F^{2}\end{array}\right)\left(\xi_{2}-\eta_{2}\right)=0$ 
and because of observability of $(h, F)$ this holds if and only if $\xi_{2}=\eta_{2}$. The system is observable since the only indistinguishable states are $\xi=\eta$.

(Necessity) Necessity of the observability of the subsystems is obvious. Necessity of $0 \notin \mathscr{P}_{1} \cap \mathscr{Z}_{2}$ is given for the particular case. We show that $0 \in \mathscr{P}_{1} \cap \mathscr{Z}_{2}$ leads to existence of indistinguishable states $\xi^{*} \neq \eta^{*}$. Necessarily then we have that $b_{3}=0$ in Eq. (17) and without loss of generality, suppose that $b_{2} \neq 0$. From the fifth equation we have that indistinguishable states should satisfy

$\left(c J \xi_{1}\right)^{j}-\left(c J \eta_{1}\right)^{j}=0, \quad j=1, \ldots, q$.

However, we are then left with only first three equations in Eq. (17). Hence, indistinguishable states can be characterized by Eq. (18) and

$$
\left(\begin{array}{c}
h \\
h F \\
h F^{2}
\end{array}\right)\left(\xi_{2}-\eta_{2}\right)=\left(\begin{array}{c}
0 \\
-h g \\
-h F g
\end{array}\right)\left(\xi_{1}-\eta_{1}\right) .
$$

Take $\xi_{1}^{*}=0$ and $\eta_{1}^{*} \neq 0$ so that $c J \eta_{1}^{*}=0$. Eq. (19) is satisfied and from the equation we obtain the unique solution $\left(\xi_{2}^{*}-\eta_{2}^{*}\right)=v^{*}$ because $(h, F)$ is observable. The pair of states

$\xi^{*}=\left(\begin{array}{c}0 \\ \eta_{2}^{*}+v^{*}\end{array}\right), \quad \eta^{*}=\left(\begin{array}{c}\eta_{1}^{*} \\ \eta_{2}^{*}\end{array}\right), \quad \eta_{1}^{*} \neq 0, \quad \eta_{2}^{*} \in \mathbb{R}^{n_{2}}$

are indistinguishable. The particular case can be easily generalized to systems of arbitrary order.

Comment 2. With our assumption on the finite relative degree of system (6), the observability conditions become very simple (note that this implies that $b \neq 0$ and $g \neq 0$ ). Indeed, if $q$ is odd we just need to check whether

1. $(c, A)$ is observable pair,

2. $(h, F)$ is observable pair,

3. $0 \notin \mathscr{P}_{1} \cap \mathscr{Z}_{2}$.

On the other hand, if $q$ is an even integer, we need to check if

1. $(c, A)$ is observable pair,

2. $A$ is nonsingular, which implies $0 \notin \mathscr{P}_{1} \cap \mathscr{Z}_{2}$,

3. $(h, F)$ is observable pair.

This simplifies the observability test considerably since only linear algebra is needed.

Comment 3. Theorem 3 can be regarded as a "dual" statement to the complete controllability results for simple Wiener-Hammerstein system which were obtained in [12] with $q$ an odd integer. Indeed, the main result on controllability of Eq. (6) in [12] states that the connection is controllable if and only if the both systems are controllable and $0 \notin \mathscr{Z}_{1} \cap \mathscr{P}_{2}$. However, for controllability the first subsystem is assumed to be the linear block $W_{1}(z)$ and the second subsystem is the static nonlinearity in series with the second linear block. The statement for dead-beat (or null) controllability is even simpler since dead-beat controllability of subsystems always implies dead-beat controllability of the overall system. In other words, the situation $0 \in \mathscr{Z}_{1} \cap \mathscr{P}_{2}$ is allowed for dead-beat controllability (since uncontrollable zero modes are allowed). With these results, analysis of controllability and observability for several classes of Wiener-Hammerstein type models is very easy.

Example 2. Consider the system:

$$
\begin{aligned}
& x_{1}(k+1)=x_{2}(k), \\
& x_{2}(k+1)=-x_{1}(k)-2 x_{2}(k)+u(k), \\
& x_{3}(k+1)-x_{4}(k), \\
& x_{4}(k+1)=-x_{3}(k)+x_{1}^{2}(k), \\
& y(k)=x_{3}(k) .
\end{aligned}
$$

The system consists of the simple Wiener model from Example 1 and a linear system. The first subsystem was shown to be observable in Example 1. Note that we could obtain the same conclusion by using Theorem 1 directly.

We test only for observability of the second linear subsystem with the matrices:

$h=\left(\begin{array}{ll}1 & 0\end{array}\right), \quad F=\left(\begin{array}{cc}0 & 1 \\ -1 & 0\end{array}\right)$

and the observability matrix

$$
\left(\begin{array}{c}
h \\
h F
\end{array}\right)=\left(\begin{array}{ll}
1 & 0 \\
0 & 1
\end{array}\right)
$$

is nonsingular. Since $A$ is nonsingular, we have that $0 \notin \mathscr{P}_{1} \cap \mathscr{Z}_{2}$, the conditions of Theorem 3 are satisfied and the simple Wiener-Hammerstein system (6) is observable.

Example 3. Consider the following simple WienerHammerstein system:

$$
\begin{aligned}
& x_{1}(k+1)=u(k), \\
& x_{2}(k+1)=x_{3}(k),
\end{aligned}
$$


$x_{3}(k+1)=-x_{2}(k)+x_{1}^{3}(k)$,

$y(k)=x_{3}(k)$.

The system consists of the linear blocks $W_{1}(z)=1 / z$ and $W_{2}(z)=z /\left(z^{2}+1\right)$ with the nonlinearity $(\cdot)^{3}$. Both linear blocks are easily seen to be observable but in this case $0 \in \mathscr{P}_{1} \cap \mathscr{Z}_{2}$ and according to Theorem 3 the system is not observable. Let us compute the set of indistinguishable states using the algorithm we presented. The ideals $J_{k}$ are given below:

$J_{1}=\left\langle\xi_{3}-\eta_{3}\right\rangle$

$J_{2}=\left\langle\xi_{3}-\eta_{3},-\xi_{2}+\xi_{1}^{3}+\eta_{2}-\eta_{1}^{3}\right\rangle$,

$J_{3}=\left\langle\xi_{3}-\eta_{3},-\xi_{2}+\xi_{1}^{3}+\eta_{2}-\eta_{1}^{3},-\xi_{3}+\eta_{3}\right\rangle$

and the Gröbner bases for ideals $J_{2}$ and $J_{3}$ are computed. They turn out to be equal $\mathrm{GB}\left[J_{2}\right]=\mathrm{GB}\left[J_{3}\right]$, which implies that $S_{z}=V\left(J_{2}\right) \cap\{(\xi, \eta): \xi \neq \eta\}$. The set of indistinguishable states:

$$
\begin{gathered}
S_{z}=\left\{(\xi, \eta) \in \mathbb{R}^{2 n}: \xi_{3}-\eta_{3}=0 \wedge-\xi_{2}+\xi_{1}^{3}\right. \\
\left.+\eta_{2}-\eta_{1}^{3}=0 \wedge \xi \neq \eta\right\}
\end{gathered}
$$

is nonempty, since for instance the states $\xi^{*}=\left(\begin{array}{lll}1 & 1 & 1\end{array}\right)^{\mathrm{T}}$ and $\eta^{*}=(281)^{\mathrm{T}}$ belong to $S_{z}$ and are indistinguishable by any input sequence.

\section{Summary}

We presented an algorithmic observability test for general polynomial systems, which stops in finite time. The test is based on the Gröbner basis method and QEPCAD algorithm. For the class of simple Wiener-Hammerstein systems we stated much simpler necessary and sufficient observability conditions. The obtained observability test is very easy to check and requires the use of linear algebra only.

\section{References}

[1] B. Buchberger, Gröbner bases: an algorithmic method in polynomial ideal theory, in: N.K. Bose (Ed.), Multidimensional Systems Theory, D. Reidel Publishing Co, Dordrecht, 1985, pp. 184-232.

[2] G.E. Collins, Quantifier elimination by cylindrical algebraic decomposition, in: B.F. Caviness, J.R. Johnson (Eds.), Quantifier Elimination and Cylindrical Algebraic Decomposition, Springer, Berlin, 1998.

[3] G.E. Collins, Hoon Hong, Partial cylindrical algebraic decomposition for quantifier elimination, J. Symbolic Comput. 12 (1991) 299-138.

[4] D. Cox, J. Little, D. O'Shea, Ideals, Varieties and Algorithms, Springer, New York, 1992.

[5] R. Haber, L. Keviczky, Identification of nonlinear dynamic systems, in: N.S. Rajbman (Ed.), Identification and System Parameter Estimation, North-Holland, Amsterdam, 1978, pp. 79-127.

[6] R. Haber, H. Unbehauen, Structure identification of nonlinear dynamic systems - a survey of input/output approaches, Automatica 26 (1990) 651-677.

[7] T. Kailath, Linear Systems, Prentice-Hall, Englewood Cliffs, NJ, 1980.

[8] R.B. Koplon, Linear systems with constrained outputs and transitions, Ph.D. Mathematics, Rutgers, The State University of New Jersey, 1994.

[9] R.B. Koplon, E.D. Sontag, Linear systems with sign observations, SIAM J. Control. Optim. 31 (1993) 1245-1266.

[10] R.B. Koplon, E.D. Sontag, M.L.J. Hautus, Observability of linear systems with saturated outputs, Linear Algebraic Appl. 205/206 (1994) 909-936.

[11] D. Nešić, A note on dead-beat controllability of generalized Hammerstein models, Systems Control Lett. 29 (1997) $223-231$.

[12] D. Nešić, Controllability for a class of simple WienerHammerstein systems, Systems Control Lett., to appear.

[13] D. Nešić, Controllability for a class of parallelly connected polynomial systems, submitted for publication.

[14] D. Nešić, I.M.Y. Mareels, Dead-beat control of simple Hammerstein models, IEEE Trans. Automat. Control 43 (1998) 1184-1189.

[15] E.D. Sontag, Polynomial Response Maps, Springer, New York, 1979.

[16] E.D. Sontag, On the observability of polynomial systems, I: finite-time problems, SIAM J. Control Optim. 17 (1979) 139-151.

[17] E.D. Sontag and Y. Rouchaleau, On discrete-time polynomial systems, Nonlinear Anal. Theory Methods Appl. 1 (1976) 55-64. 